\title{
Early Pulsar Observations with LOFAR
}

\author{
Jason Hessels ${ }^{* \dagger}$ \\ Netherlands Institute for Radio Astronomy (ASTRON), Dwingeloo, the Netherlands \\ University of Amsterdam (UvA), Amsterdam, the Netherlands \\ E-mail: hesselseastron.nl
}

Ben Stappers, Tom Hassall, Patrick Weltevrede

University of Manchester

\section{Anastasia Alexov, Thijs Coenen}

University of Amsterdam

Joeri van Leeuwen, Vlad Kondratiev, Jan David Mol

Netherlands Institute for Radio Astronomy (ASTRON)

\section{Michael Kramer, Aris Noutsos}

Max Planck Institute for Radio Astronomy

\section{Aris Karastergiou}

University of Oxford

\begin{abstract}
This contribution to the proceedings of "A New Golden Age for Radio Astronomy" is simply intended to give some of the highlights from pulsar observations with LOFAR at the time of its official opening: June 12th, 2010. These observations illustrate that, though LOFAR is still under construction and astronomical commissioning, it is already starting to deliver on its promise to revolutionize radio astronomy in the low-frequency regime. These observations also demonstrate how LOFAR has many "next-generation" capabilities, such as wide-field multi-beaming, that will be vital to open a new Golden Age in radio astronomy through the Square Kilometer Array and its precursors.
\end{abstract}

ISKAF2010 Science Meeting

June 10 -14 2010

Assen, the Netherlands

\section{* Speaker.}

$\dagger$ These results are presented on behalf of the LOFAR Transients Key Science Project. Special thanks also go to G. Heald, J. Swinbank, J. Romein, M. Wise, and V. Gajjar for very important contributions to this work. J. Hessels is a Veni Fellow of the Netherlands Organization for Scientific Research (NWO). 


\section{Introduction}

LOFAR is a low-frequency radio interferometer that covers the $10-240 \mathrm{MHz}$ frequency range, the lowest 4 octaves of the "radio window" above the Earth's ionospheric cut-off at $\sim 10 \mathrm{MHz}$. This is a relatively unexplored range of the electromagnetic spectrum, where there is still exciting potential for serendipitous astronomical discoveries. Ultimately there will be at least 40 LOFAR stations in the Netherlands, along with at least 8 international stations in Germany, England, France, and Sweden. Each of these stations contains 48/96 Low-Band Antennas (LBAs), which cover the range $10-90 \mathrm{MHz}$, as well as 48/96 High-Band Antennas (HBAs) covering the range $110-$ $240 \mathrm{MHz}$. The relatively small frequency range occupied by FM radio broadcasts is purposely filtered out to avoid this strong interference. Each station is capable of forming multiple "station beams", which are then correlated and/or summed as necessary in a central Blue Gene P (BG/P) supercomputer in Groningen. The product of station beams times total bandwidth is $48 \mathrm{MHz}$ per station, giving LOFAR a remarkably large fractional bandwidth and field-of-view (FOV). LOFAR will be the most powerful and flexible low-frequency radio telescope ever built; arguably, it is already. It is also an important precursor to the Square Kilometer Array (SKA), by demonstrating many relevant technologies for the first time. Roughly half of the LOFAR stations are currently complete, with the remaining stations to be built in the next year.

Since the summer of 2007, the LOFAR Pulsar Working Group has been performing observations with the existing LOFAR hardware in order to commission the high-time-resolution "beamformed" modes necessary to observe pulsars, "fast transients", planets, the Sun, and flare stars (to name a few sources). A description of work on the "Pulsar Pipeline" is given in [1], also in these proceedings. In $\S 2$, we describe some of the highlights of observational results so-far achieved with LOFAR. These observations nicely demonstrate much of the functionality that will make LOFAR a powerful, ground-breaking telescope for studying pulsars and other sources that vary on sub-second time-scales. Since the official opening of LOFAR on June 12th, 2010, commissioning work in this area has only intensified as we move closer to full operations. We discuss the future prospects for pulsars and LOFAR in $\S 3$. We note that a comprehensive reference paper describing pulsar observing with LOFAR is in preparation [5].

\section{Early Pulsar Observations}

\subsection{Wide-band Simultaneous Observations}

LOFAR's wide instantaneous frequency coverage in the $10-240 \mathrm{MHz}$ range is unique and can be exploited to study the frequency dependence of pulsar emission, such as its spectrum, pulseenergy distribution, and integrated pulse morphology. Similar studies in the past have suffered from very small available bandwidths, and the need to combine non-contemporaneous data, e.g., [4].

To demonstrate LOFAR's potential in this area, we performed a set of simultaneous observations in which we observed 6 bright pulsars using two LOFAR stations - one set to use the LBAs, the other set to use the HBAs - in conjunction with the 74-m Lovell Telescope at Jodrell Bank and the 100-m Effelsberg Telescope. This unique combination of telescopes allowed us to simultaneously observe the radio light from these pulsars across wavelengths from only 3.5 centimeters 
( $8.5 \mathrm{GHz})$ up to 7 meters $(43 \mathrm{MHz})$ - a factor of 200 difference. This set a new world record in simultaneous wavelength/frequency coverage for pulsars. Shown in Figure 1 are the multi-frequency pulse profiles for one of the pulsars, B1133+16.

In the future we plan to use sub-arrays ${ }^{1}$ of the LOFAR stations in order to completely cover the $10-240-\mathrm{MHz}$ frequency window simultaneously. As each station can provide up to $48 \mathrm{MHz}$ of instantaneous bandwidth, 5 sub-arrays are required for complete spectral coverage.

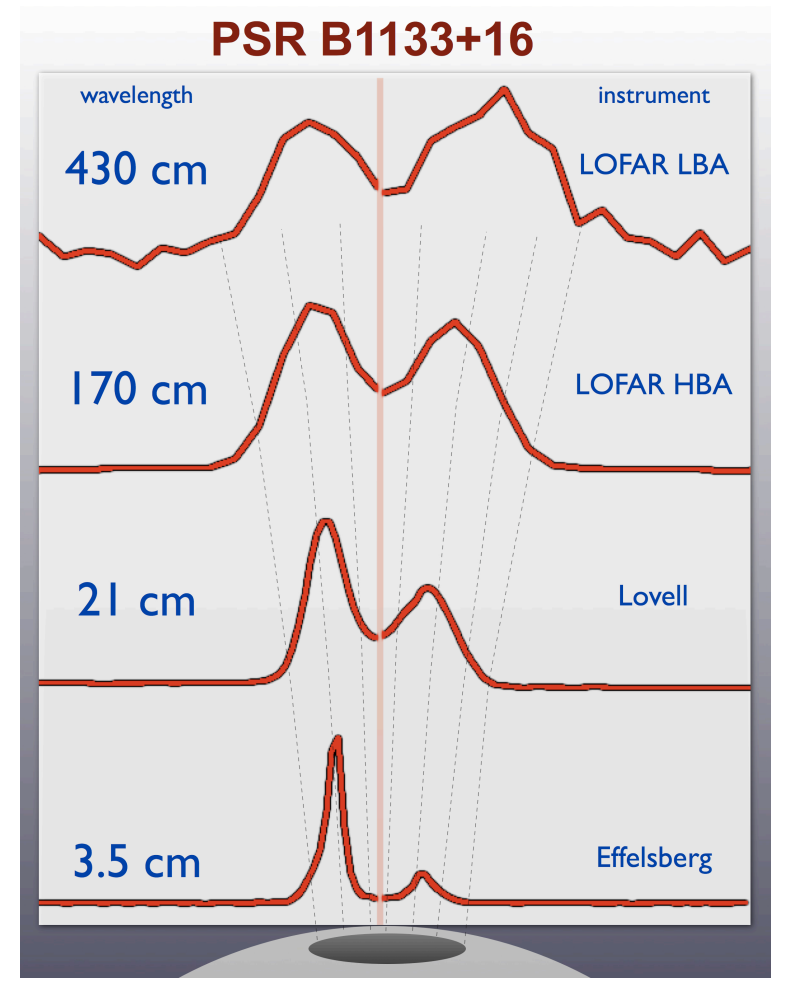

Figure 1: Simultaneous detection of pulses from PSR B1133+16 in four widely spaced bands, using the Effelsberg telescope at $3.5 \mathrm{~cm}$ wavelength $(8.5 \mathrm{GHz})$, the Lovell telescope at $21 \mathrm{~cm}$ wavelength $(1.4 \mathrm{GHz})$, and LOFAR HBAs and LBAs at $170 \mathrm{~cm}$ and $430 \mathrm{~cm}$ wavelength $(176 \mathrm{MHz} / 70 \mathrm{MHz})$, respectively. For each of the four bands, the figure shows the cumulative pulse intensity over this 1-hr observation as a function of rotational phase. In the simplest model, the shape of the pulsar's pulsed emission maps the spreading of magnetic field lines above the pulsar's magnetic poles, also depicted schematically.

\subsection{Millisecond Pulsars}

At low radio frequencies, interstellar scattering strongly affects the maximum achievable time resolution of observations because multi-path propagation of the signal causes it to be significantly smeared in time. The short ( $\sim 100 \mu \mathrm{s})$ pulses from millisecond pulsars are easily affected by this, but despite this it is still possible to observe a large fraction of the known population of millisecond pulsars using LOFAR [6]. As very few studies have been done on the low-frequency emission

\footnotetext{
${ }^{1} \mathrm{~A}$ sub-array is simply a collection of LOFAR stations configured to run in the same observing mode and pointing in the same direction. It is possible to run multiple observations simultaneously such that groups of LOFAR stations can be running each in a separate mode, at the same time.
} 
properties of millisecond pulsars, this is a potentially fruitful avenue of research. Past studies have indicated that millisecond pulsars may behave differently in this frequency regime than their more slowly rotating, higher-magnetic-field brethren ([6] and references therein).

We have detected a number of millisecond pulsars with LOFAR, including the famous 6.2-ms "Planet Pulsar" B1257+12. Figure 2 shows a diagnostic plot of this detection using the PRESTO software suite ${ }^{2}$. This was done using an incoherent dedispersion method with a large number of spectral channels. Soon we will be dedispersing these data coherently in order to much better remove the dispersive effects of the interstellar medium and to maintain the maximum time resolution available in the system $(\sim 5 \mu \mathrm{s})$.
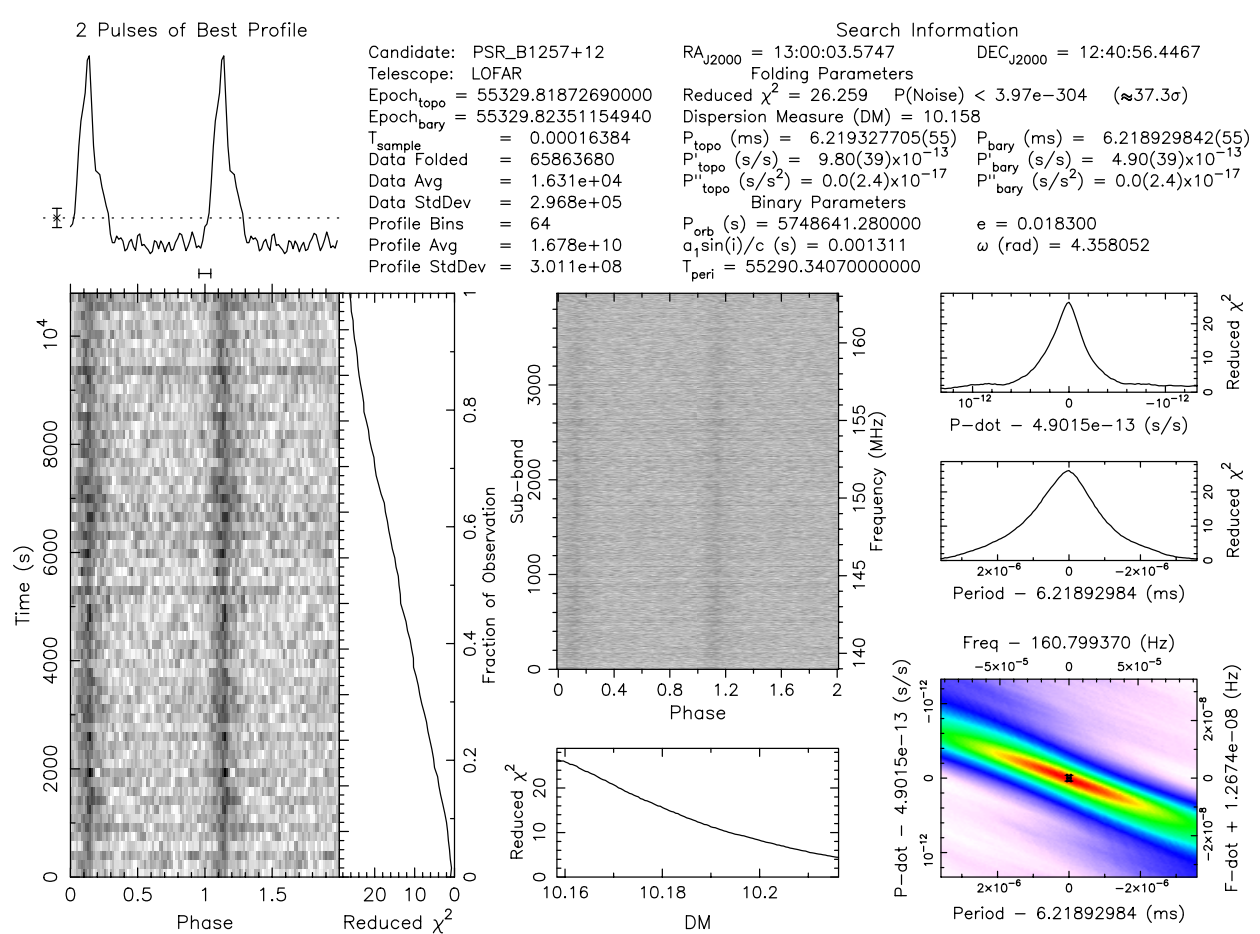

Figure 2: A diagnostic plot from the PRESTO pulsar software suite, which shows the clear detection of pulsations from the "Planet Pulsar" B1257+12. This pulsar is known to be orbited by at least 2 planetary mass bodies - the first to be discovered outside of the solar system [7]. The left-most plot shows the pulse intensity as a function of observing time, with the cumulative pulse profile (repeated twice for clarity) shown at the top. The central plot shows the signal strength across the $24 \mathrm{MHz}$ bandwidth, which was divided into $\sim 4000$ channels. At center bottom, the signal to noise is shown as a function of trial dispersion measure. Note how sensitive the signal strength is to the assumed dispersion measure. At LOFAR observing frequencies, we will likely have to adjust the dispersion measure slightly on a per epoch basis because of small changes in our line-of-sight to the pulsar with time. As can be seen here, the dispersion measure at the time of this observation deviates subtlely from the catalog value of $10.1655 \mathrm{pc} \mathrm{cm}^{-3}$. The right hand sub-panels show an optimization of the signal strength on trial period and period derivative. Observation meta-data and folding parameters are shown at the top of the plot.

\footnotetext{
${ }^{2}$ http://www.cv.nrao.edu/ sransom/presto/
} 


\subsection{Multi-Station Observations}

Pulsar observations are always sensitivity limited, and combining the signals from many LOFAR stations is critical for improving sensitivity. Ideally we will combine the stations "coherently", with a proper phase calibration - taking care of geometrical, instrumental, and environmental effects - to form small tied-array beams on the sky. So far, most multi-station LOFAR pulsar observations have combined the stations incoherently, by simply summing the station powers with the appropriate geometrical delays. Figure 3 shows data from the incoherent combination of 12 Dutch stations.

These are some of the highest quality data we have taken thus far. In particular, we note that even in the densely populated Netherlands we have been able to manage and excise the majority of the radio frequency interference by blanking only about $5 \%$ of the spectral channels. The future holds even more promise: with the full set of calibrated LOFAR stations added coherently, we should be able to achieve another factor of 10 increase in sensitivity compared with these detections.

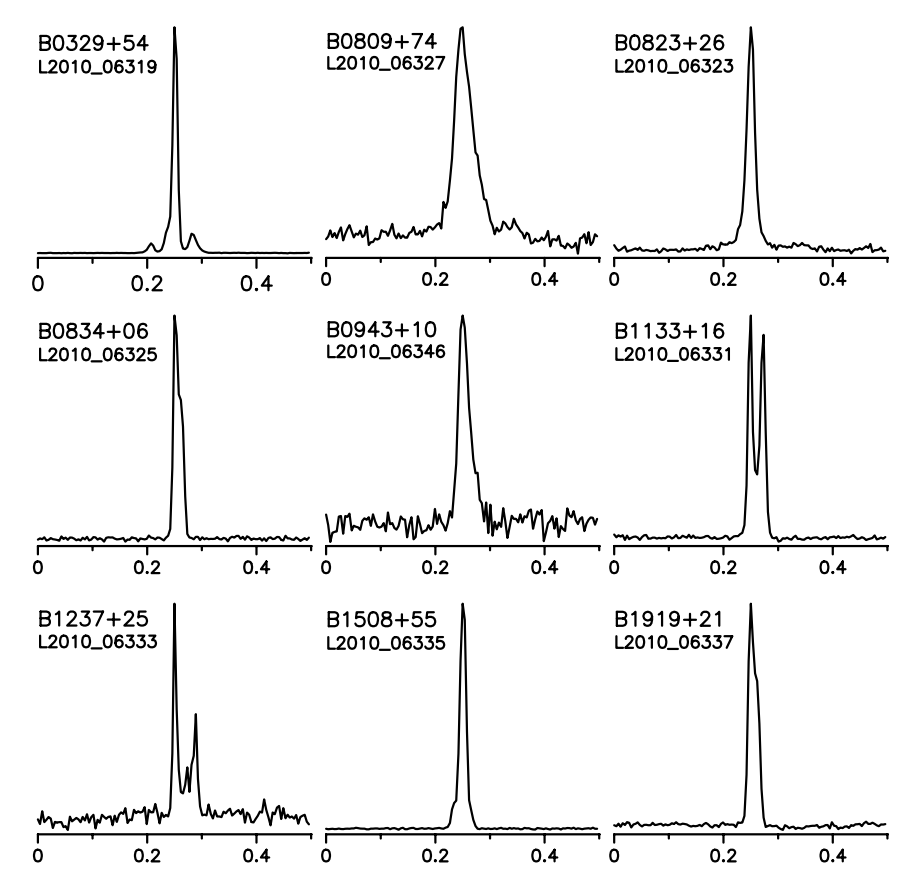

Figure 3: Pictured are the average pulse profiles of nine pulsars, observed over the course of a weekend using an automatic scheduler. Only half the rotational phase is plotted in each case in order to show finer features of the pulse morphology. Each pulsar was observed for roughly an hour with the HBA tiles (see accompanying observation ID), using different combinations of stations, with up to 12 stations at a time added incoherently.

\subsection{Simultaneous Imaging and Pulsar Observations}

On April 9, 2010, we took a 12-hr observation in which we simultaneously ran the imaging and pulsar observing modes on the LOFAR BG/P correlator. This 12-hour observation used the HBAs of 7 core and 3 remote stations from 130-178 MHz. 
The imaging data were processed using the Standard Imaging Pipeline, using the three brightest sources in the field for calibration, and excluding short baselines. Following pipeline processing, an updated sky model was derived from the image and used for a second pass of calibration; the individual subbands were processed separately but then combined before imaging into one large measurement set, so that the deconvolution could be done properly. The Common Astronomy Software Applications package $\left(\mathrm{CASA}^{3}\right)$ was used for the imaging. The resulting combined image is shown in Figure 4. The location of pulsar B0329+54 is indicated by the arrow; the pulsar has a known flux of $\sim 1 \mathrm{Jy}$ at these frequencies. The full field is very large: roughly $5^{\circ}$ across.

The simultaneously acquired, beam-formed, time series data, with a time resolution ${ }^{4}$ of $1.3 \mathrm{~ms}$, was processed using the Known Pulsar Pipeline. A small subset of roughly 200 single pulses is shown in the "pulse stack" on the right of Figure 4, with the corresponding cumulative pulse profile at the top.

This "piggy-backing" mode will be a key aspect of LOFAR operations, as it optimizes the use of available telescope time. It is particularly interesting for monitoring the sky for rare "fast transient" events and will be used along with interferometric imaging as part of the LOFAR "Radio Sky Monitor", which will regularly monitor the sky to detect transients on time-scales of milliseconds to years [2].

\subsection{Multiple Station Beams}

Unlike most conventional radio telescopes, LOFAR stations can look in multiple directions at the same time by distributing the total observing bandwidth between multiple "station beams" (FOVs) simultaneously. This makes it possible to monitor a large fraction of the sky at once and to observe multiple known sources during one observation. As such, the prospects for all-sky surveys and monitoring for rare transient events are very exciting. For the LBAs, a particular station can form beams anywhere in the visible sky; for the HBAs a preceding level of analog beam-forming at the level of the individual HBA tiles limits the forming of station beams to within the roughly $20^{\circ}$-wide FOV of the tile beam, which itself can be pointed in any desired direction.

To demonstrate this impressive functionality, we observed two widely separated pulsars simultaneously. Pulsars B0329+54 and B0450+55 are separated by close to $12^{\circ}$ on the sky, or, to put it another way, by roughly 24 times the width of the full moon (Figure 5). Pointing the $20^{\circ} \mathrm{HBA}$ tile beam directly between the two pulsars, we formed two station beams and centered one on each of the two pulsars. Tracking the sources for $30 \mathrm{~min}$, both the very bright B0329+54 (the brightest pulsar in the northern sky) and the much fainter B0450+55 were clearly detected.

We plan to repeat this type of observation using more beams on more sources. With the LBAs, each of which can effectively see the whole sky above the horizon, it will be possible to form station

\footnotetext{
${ }^{3}$ http://casa.nrao.edu/

${ }^{4}$ For ease of processing, these data were heavily downsampled in time online. Much higher time resolution, up to $5.12 \mu \mathrm{s}$ - limited by the $195.3-\mathrm{kHz}$ subbands that are sent from the stations - is achievable with the current system. In the future, an inverse poly-phase filter on the station subbands may allow much higher time resolution still (down to $10 \mathrm{~ns}$ ), which will be used for detecting radio emission from the wake of cosmic rays entering the Earth's atmosphere. Such high time resolution also has applications for the study of pulsar giant pulses, though scattering remains a significant limiting factor on the effective time resolution achievable at these observing frequencies.
} 


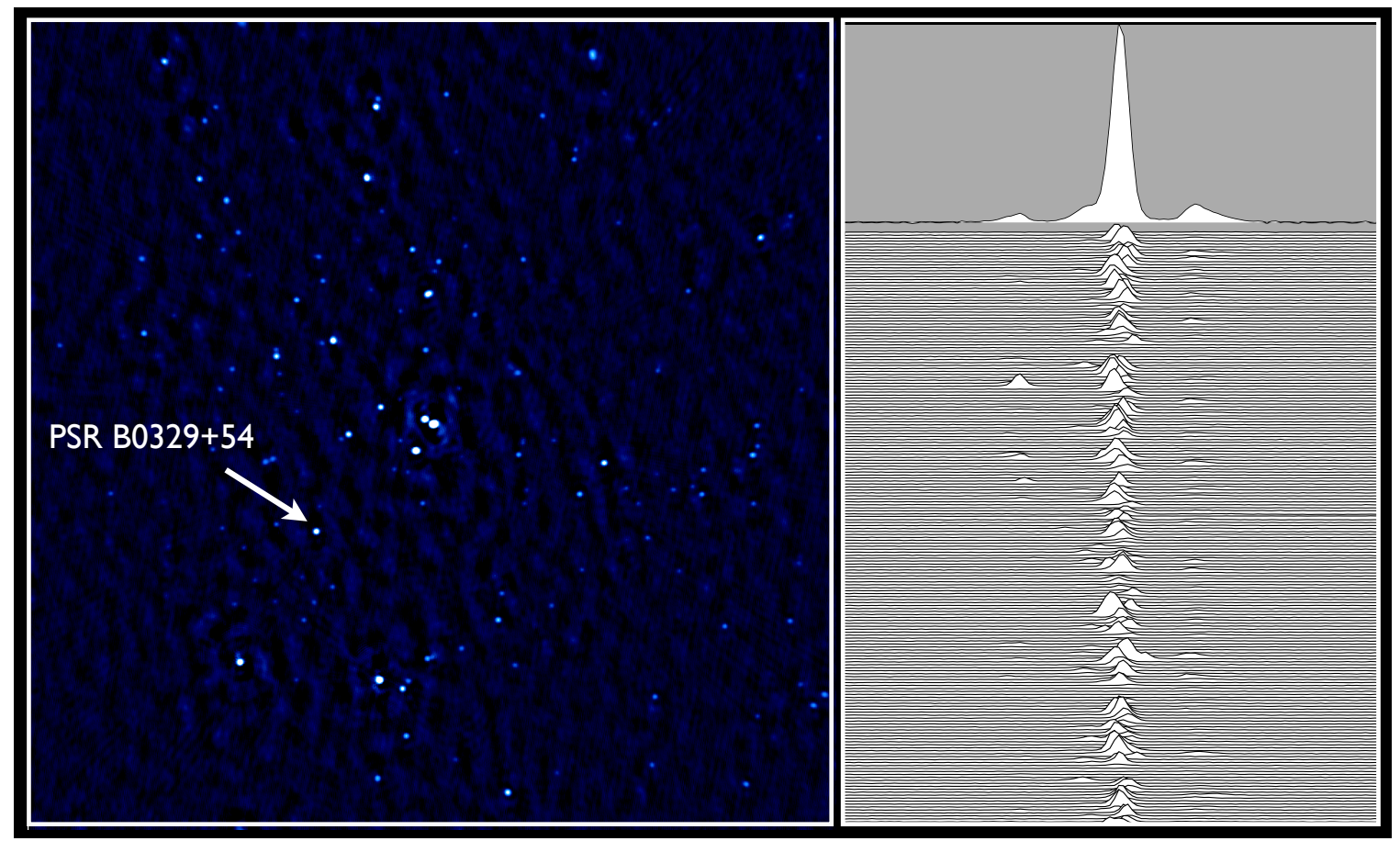

Figure 4: Left: A LOFAR HBA image of a $5^{\circ} \times 5^{\circ}$ field containing the very bright pulsar B0329+54. This pulsar has a flux density of roughly $1 \mathrm{Jy}$ at $150 \mathrm{MHz}$ and is one of the brighter sources in the field. Right: Pulse stack of roughly 200 pulses from the same imaging/beam-formed observation. Significant pulse-topulse profile morphology and intensity variations are obvious. The sum of these pulses is shown at the top.

beams that are even more widely separated. To do something similar with the HBAs would require forming sub-arrays of the LOFAR stations, akin to a "Fly's Eye" mode.

\subsection{Crab Giant Pulses}

The Crab pulsar is the proto-type of a small population of radio pulsars known to emit short, extremely bright "giant pulses". These pulses can intrinsically be as short as nanoseconds or less [3], and have the highest brightness temperatures of any known astrophysical phenomenon. At low radio frequency, the Crab pulsar's giant pulses are severely scattered, creating a long exponential tail on their trailing edge. As an example, Figure 6 shows a heavily scattered giant pulse detection with the LOFAR HBAs $\left(v_{\text {center }} \sim 160 \mathrm{MHz}\right)$. From this figure, it can been seen that the length of the pulse is actually scattered to a time-scale longer than the pulse period (the cumulative pulseprofile is shown in the background). Interestingly, this particular pulse appears to be a double giant pulse, with two such pulses occurring separated by only one pulse period (this is not the interpulse). Similar detection techniques will be used to search for bright, dispersed pulses from extragalactic pulsars and even potential cosmological bursts.

In the future, observations of known sources like the Crab will greatly benefit from the formation of tied-array beams (see §3), which will restrict the FOV and hence reduce the contribution of background emission (in this case, the very bright Crab Nebula). 


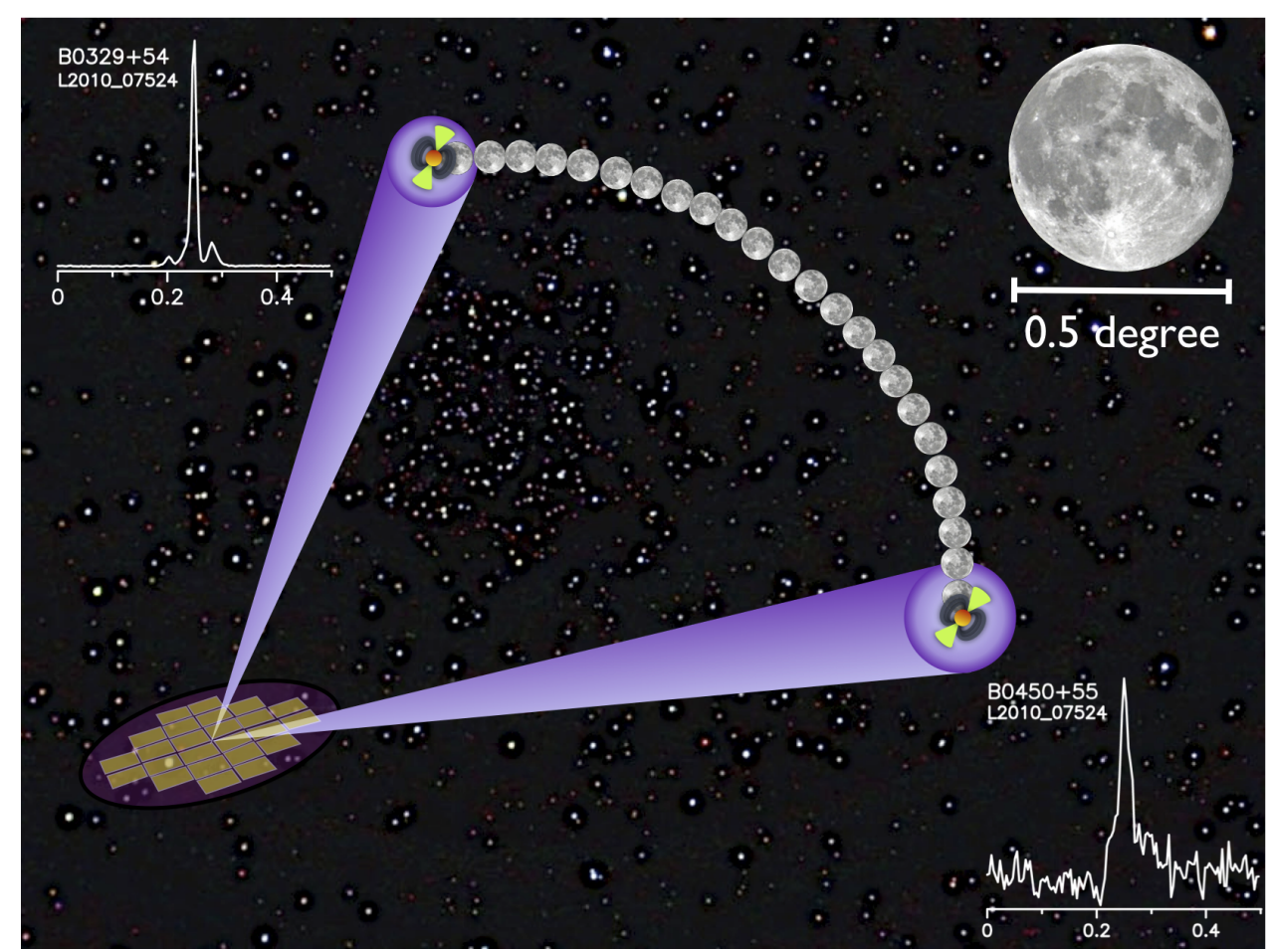

Figure 5: An artist's impression of the simultaneous detection of pulsars B0329+54 and B0450+55 using two station beams. These pulsars are separated by close to $12^{\circ}$ on the sky, roughly 24 times the width of the full moon.

\subsection{Single Station "Fringes"}

Pulsar observations are not only an excellent way to test LOFAR's beam-formed modes; they are also well-suited for more generic commissioning tests of the system. As an example, we have taken good advantage of the fact that the brightest pulsar in the northern hemisphere, PSR B0329+54, fortuitously passes through the Zenith at the latitude of the LOFAR core in Exloo, the Netherlands. This has permitted numerous tests that use the fact that the pulsar will drift through the station beam of elements that are added with zero geometrical delay. This has been used, e.g., to successfully debug pointing and tracking problems in the past.

A particularly nice example is a transit observation in which PSR B0329+54 passed through the summed beam of the two HBA "ears" of core station CS302. Each core station has two sets of 24 HBA tiles separated by roughly $130 \mathrm{~m}$ in order to provide a wealth of short baselines for imaging. When combined coherently, the resulting, instantaneous beam should form a fringe pattern of fan beams. To test that this was indeed the case, we observed a transit of PSR B0329+54 in this mode. Figure 7 gives an artist's impression of this setup and the red rectangle shows the folded pulse profile as a function of rotational phase and observing time. As the pulsar passes through the fan beams, it's observed strength is seen to rise and fall in accordance with the expected beam pattern. 


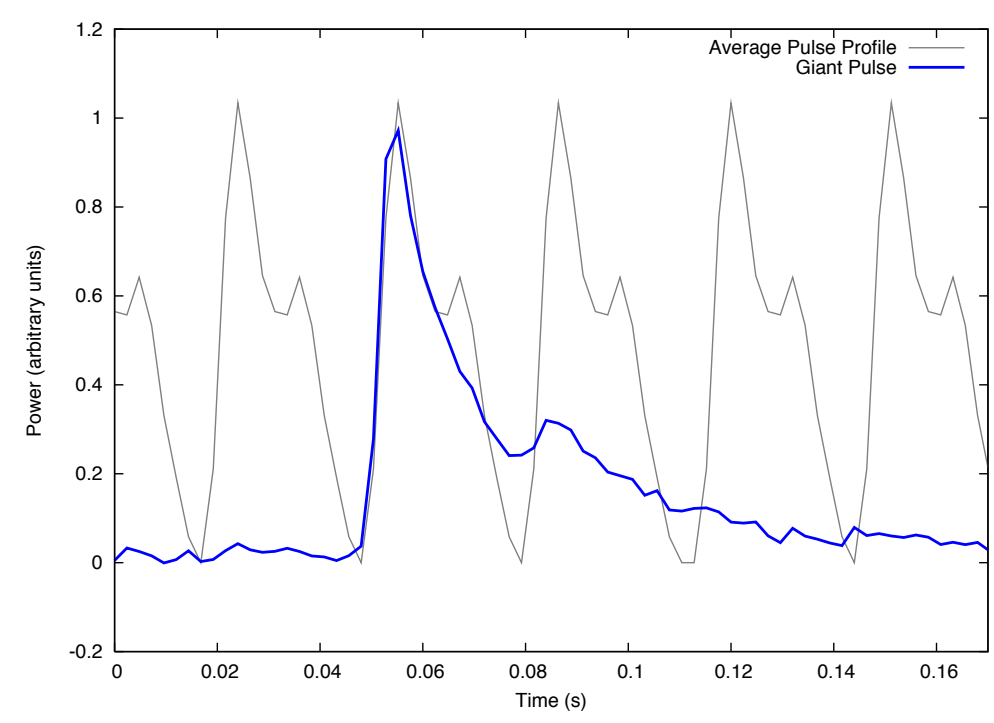

Figure 6: Example giant pulse as observed with the LOFAR HBAs at a central frequency of $160 \mathrm{MHz}$. Interestingly, this particular pulse appears to be a double giant pulse, with two such pulses occurring separated by only one pulse period (this is not the interpulse).

\section{Conclusions and Future Prospects}

As shown here, commissioning of LOFAR for pulsar observations has made great strides in the last year. The ability to simultaneously image and record at high time resolution coupled with LOFAR's proven multi-beaming capabilities will make it a powerful instrument for exploring the transient radio sky.

However, despite the many successful observations we have presented here, there remains much commissioning work still to be done. In fact, it is likely that several years from now we will still be improving the observational setup in order to reap the maximum benefit of LOFAR's hardware. In the meantime, however, we hope that increasingly impressive observational results continue to be achieved. Since the official LOFAR opening, work on the beam-formed modes has continued in earnest. Here we comment on this current and future work.

Automated Observing: The debugging of automated observing pipelines, so that the modes that are already working can be used to observe regularly and efficiently, is now well under way. In late July and early August 2010, we have done several all-weekend campaigns with hundreds of $10-30$-min observations of known pulsars. Currently the down-time between successive observations is 3 minutes - still impressive compared with a conventional telescope that is steered mechanically - and is expected to improve. These observations have allowed us to clearly detect over 100 known pulsars; their wide-band, low-frequency pulse profiles will be presented in a future paper. These data also offer the opportunity to exercise our proto-type search pipeline, which has already been successful at detecting even faint, known pulsars and with managing radio frequency interference excision. An (expected) consequence of LOFAR's complex beam pattern and sidelobes is that the very brightest pulsars $(>100 \mathrm{mJy}$ at $150 \mathrm{MHz})$ can be detected very far from their true direction. In the extreme case, we have even detected PSR B1508+55 in a beam pointed $64^{\circ}$ 


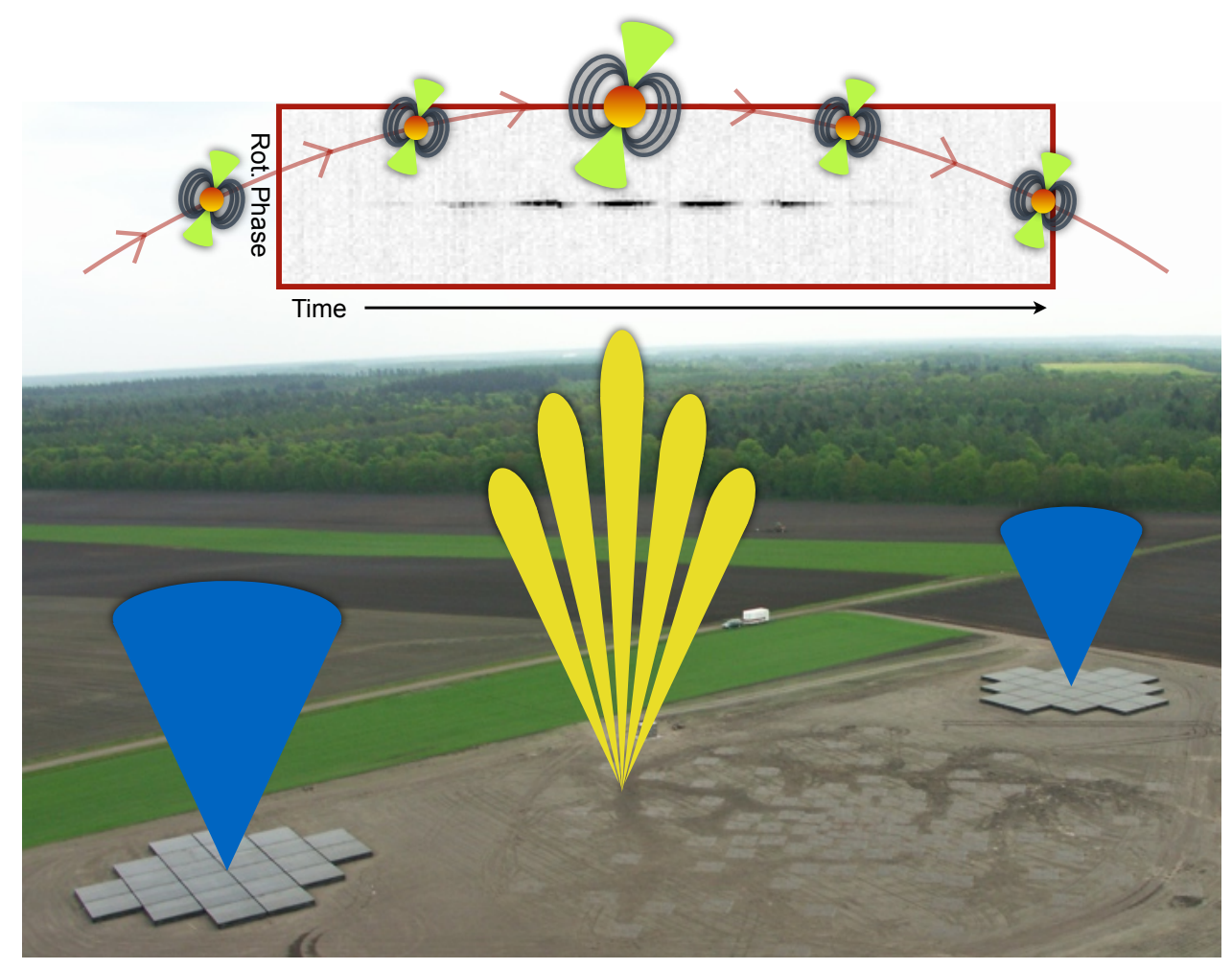

Figure 7: Artist's impression of the very bright pulsar B0329+54 as it transits through the Zenith. The red rectangle shows the actual data: the folded pulse profile as a function of rotational phase and observing time. As the pulsar passes through the fan beams, it's observed strength is seen to rise and fall in accordance with the expected beam pattern.

away from the pulsar's true position. This effect presents an additional challenge in pulsar surveys.

Coherent Dedispersion: Especially for millisecond pulsars, coherent dedispersion will be required. It is possible to write out the LOFAR station data as complex samples, which allows us to then apply coherent dedispersion to the data offline. A rudimentary version of this pipeline is now working and the results are encouraging. Ultimately, it is our plan to implement this online on the BG/P correlator so that observations of known pulsars can have the benefit of coherent dedispersion without the burden of recording with large data rates.

Tied-Array Beams: To form proper tied-array beams requires a precise calibration and correction of geometrical, instrumental, and environmental phase delays between stations. The geometrical delays are of course the most straight-forward and are routinely applied when we form incoherent summations of the station signals. The spring and summer of 2010 has seen much work dedicated to understanding and correcting the instrumental phase delays between stations. In particular a new "Single Clock" was installed near the site of the LOFAR "Superterp", which is the central core of the array, containing the 6 innermost LOFAR stations. This Single Clock provides a common clock signal to these stations, which removes the need to calibrate the clock drifts between stations. It is possible that this could be extended to other nearby stations in the LOFAR core. Early tests of the Single Clock have been very encouraging, and we now believe we are close to combining all the 6 Superterp stations coherently. As these stations are within a circular diameter 
of only about $350 \mathrm{~m}$, there is likely no need for further calibration of differential ionospheric phase delays between the stations. Proper correction for the ionosphere remains the greatest challenge to forming tied-array beams with all core stations, and we are currently investigating calibration schemes that make use of LOFAR's ability to simultaneous image and take pulsar-like data.

\section{References}

[1] A. Alexov et al., The LOFAR Known Pulsar Data Pipeline, in proceedings of The ISKAF2010 Science Meeting, 2010, POS (ISKAF 2010) 060.

[2] R. Fender et al., LOFAR Transients and the Radio Sky Monitor, in proceedings of Bursts, Pulses and Flickering: wide-field monitoring of the dynamic radio sky, Tzioumis, Lazio \& Fender (Eds), Proceedings of Science, 2007.

[3] T.H. Hankins et al., Nanosecond radio bursts from strong plasma turbulence in the Crab pulsar, Nature, 2003, Volume 422, Issue 6928, pp. 141-143.

[4] O.I. Malov \& V.M. Malofeev, Average pulse profiles of radio pulsars at 102 and $111 \mathrm{MHz}$, Astronomy Reports, 2010, Volume 54, Issue 3, pp. 210-227.

[5] B.W. Stappers et al., Pulsars \& LOFAR, University of Manchester, 2011 in prep.

[6] B.W. Stappers et al., Low Frequency Observations of Millisecond Pulsars with the WSRT, in proceedings of 40 YEARS OF PULSARS: Millisecond Pulsars, Magnetars and More, 2008, AIP Conference Proceedings, Volume 983, pp. 593-597.

[7] A. Wolszczan \& D.A. Frail, A planetary system around the millisecond pulsar PSR1257+12, Nature, 1992, vol. 355, pp. 145-147. 